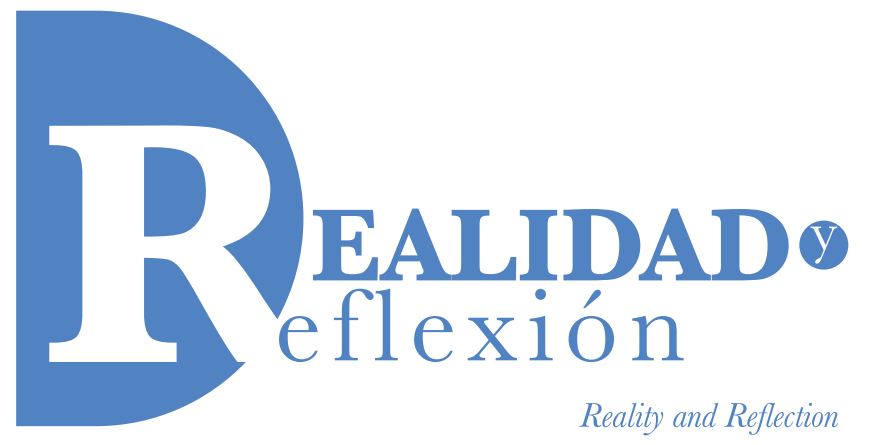

ISSN 1992-6510

e-ISSN 2520-9299

Año 18, N 47, San Salvador, El Salvador, Centroamérica. Revista Semestral Enero-Junio 2018

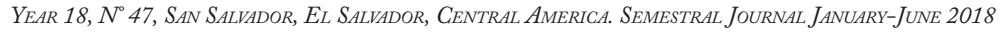

\title{
Hacia un manejo sostenible de pesquerías en El Salvador
}

\section{Towards Sustainable Fisheries Management in El Salvador}

\author{
Enrique Patiño Burch \\ Director Ejecutivo de ProPesca \\ Licenciatura en Ciencias Pesqueras, Oregon State University \\ Maestría en Ciencias Acuicultura, Auburn Unveristy \\ Biólogo Pesquero con El Servicio Nacional de Pesquerías de los Estados Unidos (2000-2015) \\ Biólogo pesquero. Consultor con El Servicio Nacional de Pesquerías de los Estados Unidos (2016-2018) \\ enriquep@propesca.org \\ Recibido: 15 de marzo de 2018 \\ Aprobado: 22 de mayo de 2018 \\ DOI: http://dx.doi.org/10.5377/ryr.v0i47.6276
}

\section{RESUMEN}

El Salvador necesita mejorar la forma en que administra y regula sus actividades pesqueras como país, si lo que se pretende es cumplir con los objetivos de sus propias políticas y con sus compromisos regionales e internacionales. Se requiere un nuevo enfoque y un cambio de paradigma. Los principales obstáculos que deben superarse son de índole político. Este artículo examina los conceptos, principios y elementos necesarios para entender lo que significa lograr pesquerías sostenibles en El Salvador, también, propone una reforma institucional basada consideraciones técnicas.

Palabras clave: Environmental Protection Index, gobernanza, institucionalidad, normativa, pesquerías sostenibles, política.

\section{ABSTRACT}

El Salvador needs to improve how it administers and regulates its fisheries activities as a country, if the country wants to comply with the objectives of its own policies and with its regional and international obligations. What is needed now is a new focus, along with and a change in paradigm. The current obstacles to the needed changes are political in nature. This article examines the concepts, principles and elements necessary to understand it means to achieve sustainable fisheries in El Salvador, and also proposes an institutional reform based on technical considerations.

Keywords: Environmental Protection Index, governance, institutionality, law, policy, sustainable fisheries, political. 


\section{Introducción}

$\mathrm{El}$ potencial que tienen la pesca y la acuicultura para aportar al bienestar social y económico a largo plazo depende de la sostenibilidad de las mismas. Lograr pesquerías sostenibles es actualmente un desafío global. Las prácticas no sostenibles continúan siendo un peligro para la biodiversidad, la seguridad alimentaria y los medios de subsistencia de millones de personas. El objetivo de este artículo es justificar una reforma institucional con el propósito de que en el futuro, como país, El salvador logre manejar sus pesquerías de forma sostenible.

De acuerdo a Costello et al. (2012), habrían importantes beneficios ecológicos y de alimentación a nivel mundial con un mejor manejo de las pesquerías. Pero para realizar estos beneficios se requiere de reformas significativas y acertadas. Para lograr estos beneficios en países subdesarrollados como El Salvador, se requiere de buena gobernanza, aplicación y cumplimiento de las leyes y adecuados programas de investigación y monitoreo. En países subdesarrollados, a corto plazo, el uso de zonas exclusivas de pesca, el cooperativismo y un enfoque de comanejo podrían ser los instrumentos más apropiados.

Entender el beneficio social y económico para el país asociado con lograr pesquerías sostenibles no es el enfoque de este artículo. E1 enfoque de este artículo es técnico en el sentido que se enfoca en lo que se requeriría para que en El Salvador las pesquerías sean manejadas de forma sostenible. Primero se articulan los requisitos para una adecuada gestión, para luego proponer una forma de reestructuración institucional orientada en lograr manejar las pesquerías nacionales de forma sostenible.

Este artículo pretende contribuir al debate nacional, contribuyendo en informar al público, a los políticos actuales y futuros, y a funcionarios gubernamentales. Con un inminente cambio en la administración publica en el país (alcaldes y diputados en el 2018 y la presidencia en el 2019), se hace necesario intentar de nuevo dimensionar el desafío que tiene el país y así entender la dirección que hay que tomar.

\section{1. ¿Cómo entender pesquerías sostenibles en El Salvador?}

Hay varias formas de abordar el tema de pesquerías sostenibles. De cualquier forma que uno lo vea, lograr y mantener sostenibilidad en las pesquerías requiere de un continuo flujo de información pertinente y actualizada, transparencia en el uso de esta información, participación de los sectores en la toma de decisiones, capacidad técnica, científica $\mathrm{y}$ económica, y voluntad política.

Ordenación Pesquera, según la guía del administrador pesquero (FAO 2005), es "el proceso integrado de recolección de información, análisis, planificación, consulta, adopción de decisiones, asignación de recursos y formulación y ejecución, así como imposición cuando sea necesario, de reglamentos o normas que rijan las actividades pesqueras para asegurar la productividad de los recursos y la consecución de otros objetivos". El objetivo de una ordenación pesquera es garantizar la sostenibilidad de las pesquerías. 
Otra manera de pensar en cómo lograr pesquerías sostenibles es viéndolo desde el punto de vista de "gobernanza" pesquera, examinando el "sistema pesquero". En este sistema, la actividad pesquera puede visualizarse como el resultado de tres componentes que interactúan y que son interdependientes: la política, la normativa y la institucionalidad.

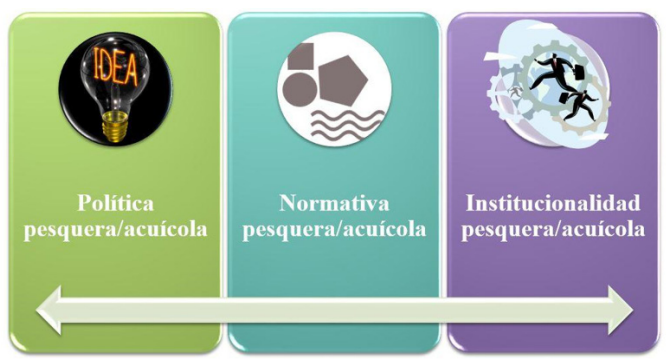

Figura $n .^{\circ}$ 1. El sistema pesquero: la política, la normativa y la institucionalidad.

Según la FAO (2004), "el sentido y alcance de estos componentes, distintos pero inseparables, configura una red sistémica entendida como un conjunto de elementos entrelazados que pueden obrar a favor o en contra del desarrollo sostenible, según sean o no adecuadamente manejados”. Es decir, como país habrá que enfocarse en asegurar que cada uno de estos elementos obre a favor de lograr pesquerías sostenibles.

En El Salvador, la Política Nacional de Pesca y Acuicultura 2015-2030 (MAG, 2015a) es un buen instrumento, pero es muy distinta a la política que se implementa en el terreno. No se han hecho los reajustes necesarios en funciones institucionales y en las prácticas del trabajo institucional para responder a la nueva política. La actual institución no tiene la capacidad de implementar su propia política. Tampoco se sabe si la próxima administración del Gobierno central continuará con la actual política nacional o elaborara una nueva política.

En El Salvador, la normativa pesquera vigente no refleja los objetivos de la Política Nacional de Pesca y Acuicultura 2015-2030. El eje estratégico número uno de la política nacional habla de una reforma para un fortalecimiento institucional. La ley vigente no obliga este cumplimiento. El eje estratégico número dos de la política nacional llama a fortalecer el enfoque científico y de capacidad de investigación de la institución. La ley vigente no garantiza encaminar a la institución en esa dirección. La normativa necesita ser actualizada de tal forma que garantice el cumplimiento de los objetivos, de acuerdo a los principios de la política nacional de pesca vigente. Simplemente no se han visualizado los cambios necesarios en la normativa en relación a sus ejes estratégicos para adaptar la estructura y desempeño de la institución de acuerdo a los objetivos y principios de la nueva política del 2015.

En E1 Salvador, la institucionalidad pesquera es deficiente, donde la investigación y la innovación son muy pobres, donde no existe transparencia o participación de los sectores en la toma de decisiones, donde las pesquerías operan de forma desordenada y con muy poco control. Las leyes, el manejo, el control y fiscalización, la investigación y lo académico son muy débiles. La cultura y la educación nacional alrededor de los recursos pesqueros también es deficiente, y la sociedad contribuye muy poco para lograr que las pesquerías nacionales se manejen de forma sostenible. Hay contaminación de todo tipo y un consumo indiscriminado de productos 
pesqueros, por ejemplo, y existe una carencia de presión social para obligar que los cambios necesarios en el Estado y la sociedad se den de acuerdo a la política y a la normativa.

En El Salvador, una gestión enfocada en lograr la sostenibilidad de sus pesquerías significaría un reordenamiento en las funciones de todas las instituciones de Gobierno que tengan cualquier tipo de jurisdicción en temas de administración, reglamentación, fiscalización, promoción, etc., de actividades pesqueras de tal forma que el Estado garantice el cumplimiento del objetivo general de la Política Nacional de Pesca y Acuicultura 2015-2030. Significaría un nuevo y serio enfoque científico y académico, además de transparencia en la diseminación de información y con la participación de las partes interesadas en la toma de decisiones. Se requerirá adecuar el Plan de Desarrollo Sustentable de la Pesca y la Acuicultura en El Salvador (MAG 2016) y particularmente el Plan Nacional de Ordenación Pesquera 2015-2030 (MAG 2015b) de acuerdo a un nuevo enfoque y a un nuevo paradigma. Se requerirá desarrollar planes de manejo para cada pesquería. Actualmente no existe algún plan de manejo para alguna pesquería en el país.

A nivel internacional, los estándares de lo que significa pesquerías sostenibles están bien desarrollados y documentados. Lo mismo es cierto para índices de calidad de gestión pesquera. Por ejemplo, el Estándar de Pesquerías del Marine Stewardship Council $(M S C)^{1}$ está diseñado para evaluar si una pesquería está bien gestionada y es sostenible. Según el MSC, todas las pesquerías deben cumplir tres principios

1 https://20.msc.org/home básicos. (Ver Figura n. ${ }^{\circ}$ 2).

Según el MSC, una pesca "sustentable" (sostenible) es aquella que se lleva a cabo de manera que:

1. Se pueda continuar indefinidamente a niveles razonables.

2. Mantenga y busque aumentar al máximo la salud y la abundancia ecológica.

3. Mantenga la diversidad, estructura y función del ecosistema del cual depende así como la calidad del hábitat, reduciendo al mínimo los efectos adversos que provoque.

4. Se maneje y opere de manera responsable y conforme a las leyes y reglamentos locales, nacionales e internacionales.

5. Mantenga opciones y beneficios socioeconómicos presentes y futuros.

6. Selleveacabo demanerasocioeconómicamente justa y responsable.

Estos son básicamente los seis requisitos para una ecoetiquetación de pesquerías sostenibles por el MSC, y concuerdan perfectamente con el objetivo de la Política Nacional de Pesca y Acuicultura 2015-2030. La aplicación de planes de manejo de pesquerías de acuerdo a principios científicos y políticos es un requisito para lograr la gobernanza necesaria para manejar pesquerías de forma sostenible. Para que una pesquería se lleve a cabo de manera socioeconómicamente justa y responsable, por ejemplo, se requiere de una política que así lo indique y de acuerdos intersectoriales para evitar conflictos en la 


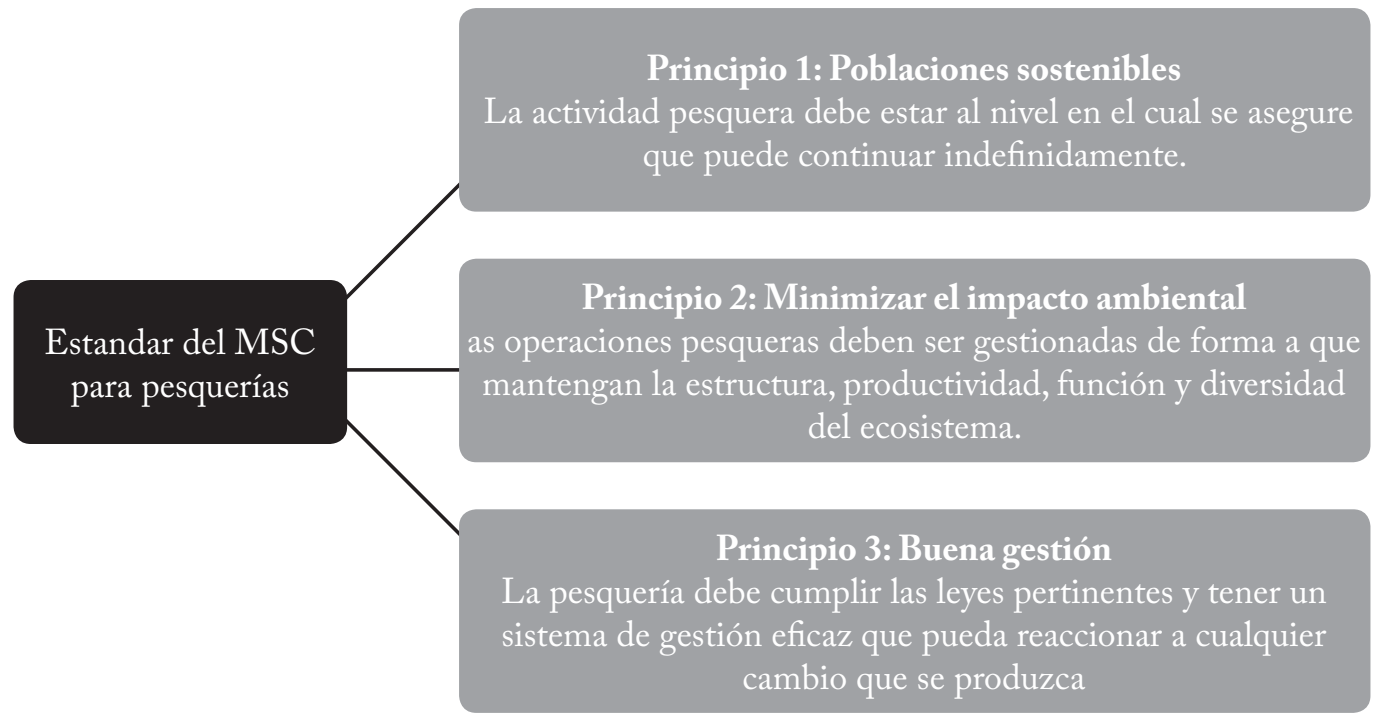

Figura n. ${ }^{\circ}$ 2. Principios Básicos de Pesquerías Sostenibles. Fuente: Marine Stewardship Council (MSC).

aplicación de normativas pesqueras. Esos seis requisitos del MSC no se cumplen para ninguna pesquería en El Salvador, y las pesquerías se desarrollan de forma no sostenible.

Una forma de evaluar la calidad en la gestión de pesquerías a nivel internacional es el Índice de Desempeño Ambiental (EPI, por sus siglas en inglés²). E1 EPI clasifica a 180 países en nueve temas ambientales prioritarios de acuerdo a dos objetivos: la protección de la salud humana y el mantenimiento de la vitalidad de los ecosistemas. El objetivo del EPI es eliminar los argumentos emocionales y retóricos en el debate ambiental y buscar una forma de abordar los temas basada más en la evidencia y los datos, y así facilitar el seguimiento y monitoreo del desempeño y la responsabilidad de los que toman las decisiones. E1 EPI es un estándar para monitorear el desempeño

\footnotetext{
2 https://epi.envirocenter.yale.edu/

3. http://www.seaaroundus.org/data/\#/eez/222/stock-status y http:// www.seaaroundus.org/data/\#/eez/222/marine-trophic-index
}

de un país en políticas internacionales y para comparar desempeño entre países, como por ejemplo en relación a los Objetivos de Desarrollo Sostenible (ODS) de las Naciones Unidas.

En el 2018, El Salvador ocupa el lugar 106 de 180 países evaluados, con una calificación de 53.91 (Figura 3). Sin embargo, en el sector pesca, El Salvador tiene la peor calificación de todos los países evaluados y ocupa el último lugar.

El puntaje de las pesquerías para cada país, de 2018, en el EPI se basa en dos indicadores elaborados por la organización Sea Around Us: ${ }^{3}$

1. El porcentaje de la captura total de un país que proviene de taxones clasificados como colapsados o sobreexplotados

2. Tendencias en el Índice trófico marino regional, en marcos temporales de 5 y 10 años. 


\section{Perfil de País}

\section{EL SALVADOR}

2018 Rango de País (fuera de 180)

106

Puntaje $(0=$ Peor; $100=$ Mejor $)$

53.91

Puntaje de país

CATEGORIAS RANGO
Interpretación de indicadores

Medio Ambientales (EPI)

- Población (millones) 6.3

- Área de tierra $(\mathrm{km}) \quad \mathbf{2 0 , 7 2 0}$

- PIB (PPA (Paridad del poder adquisitivo, billones) $\mathbf{5 0}$

- PIB per cápita

7,990

- SDG índex. (Indicadores de metas de desarrollo sustentable) 62.9

\begin{tabular}{|l|r|}
\hline $\begin{array}{l}\text { Salud medio } \\
\text { ambiental }\end{array}$ & 88 \\
\hline Calidad de aire & 67 \\
\hline $\begin{array}{l}\text { Saneamiento y } \\
\text { agua }\end{array}$ & 109 \\
\hline $\begin{array}{l}\text { Metales } \\
\text { pesados }\end{array}$ & 139 \\
\hline $\begin{array}{l}\text { Vitalidad del } \\
\text { ecosistema }\end{array}$ & 123 \\
\hline $\begin{array}{l}\text { Hábitat y } \\
\text { biodiversidad }\end{array}$ & 107 \\
\hline Bosques & 67 \\
\hline Pesquerías & 136 \\
\hline $\begin{array}{l}\text { Clima y } \\
\text { energía }\end{array}$ \\
\hline $\begin{array}{l}\text { Contaminación } \\
\text { de aire }\end{array}$ & 154 \\
\hline $\begin{array}{l}\text { Fuentes de } \\
\text { agua }\end{array}$ & 153 \\
\hline Agricultura & 36 \\
\hline
\end{tabular}

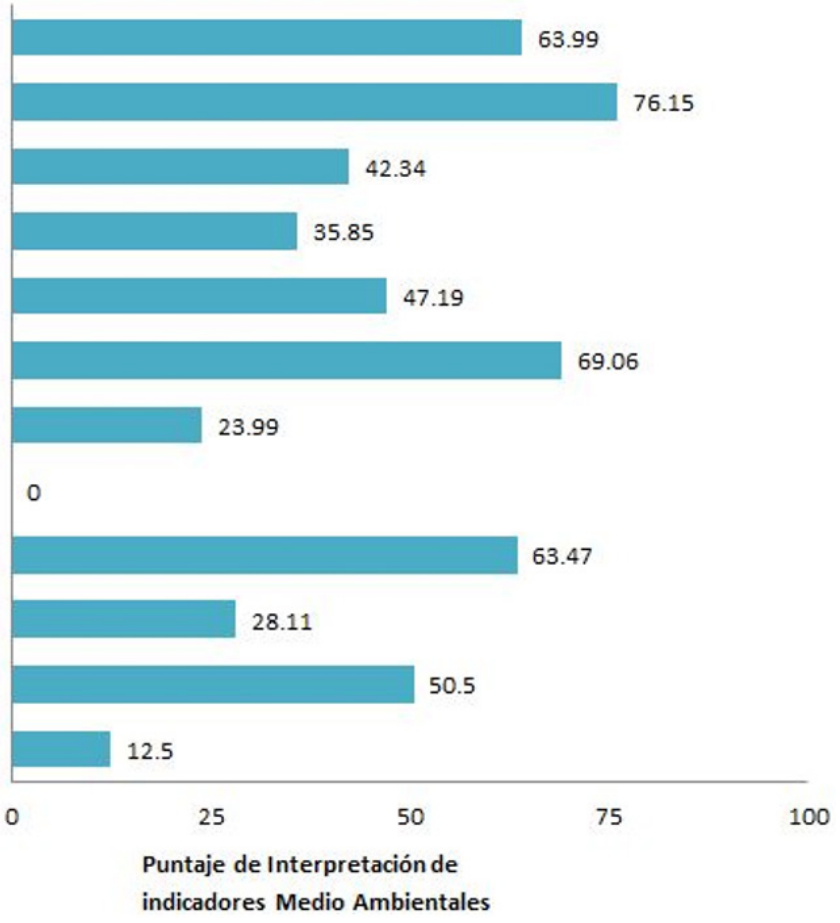

Figura n. ${ }^{\circ}$ 3. Índice de Desempeño Ambiental (Environmental Performance Index) para el Salvador. Fuente: Enviromental Perfomance Index: Country Profile E1 Salvador. 
El EPI probablemente necesita de ciertos ajustes o correcciones específicas para cada país. La información para pesquerías en el EPI es derivada de datos de la FAO, la cual le proporciona cada país. Y en el caso de E1 Salvador esta información no es confiable, especialmente desde el 2007 cuando las estadísticas pesqueras decayeron. Sin embargo esto refleja otro tipo de problema y el panorama general que presenta la Figura n. 3 podría verse como un índice de calidad de gestión. Es un índice útil en casos como en El Salvador, donde las estadísticas pesqueras son muy deficientes. Es muy probable que corrigiendo la falta de estadísticas pesqueras, el EPI para pesquerías de El Salvador mejore. Y corregir la falta de estadísticas pesqueras es consistente y necesario con la noción de lograr y mantener pesquerías sostenibles.

E1 EPI, específicamente el índice de pesquerías, es un estándar internacional para comparar y monitorear el desempeño de la gestión pesquera de un país, y El Salvador anda bastante mal con una calificación de cero puntos y una posición de último lugar de los países evaluados.

Independientemente de estos estándares e índices internacionales, es evidente que El Salvador no está obteniendo el óptimo beneficio derivado de la pesca. Cualquier indicador que se utilice para evaluar el estado actual de la gestión del sector pesca revela un panorama preocupante. Esto es debido principalmente a una inadecuada gobernanza de los recursos pesqueros. Y como la sustentabilidad de las pesquerías en cualquier lugar del mundo está en estrecha relación con la gobernabilidad de las mismas, los objetivos de la Política Nacional de Pesca y Acuicultura 2015-
2030 no se lograrán sin avances significativos hacia una adecuada gobernanza pesquera.

La Dirección General de Desarrollo de la Pesca y la Acuicultura (CENDEPESCA) es parte del Ministerio de Agricultura y Ganadería (MAG), y es la institución responsable por la administración y regulación de las actividades pesqueras en E1 Salvador. E1 Estado salvadoreño ha estado directamente involucrado en la gestión de la pesca desde los años cincuentas, y CENDEPESCA ha existido desde 1980 (López, 2017). Sin embargo, el país sigue teniendo serios problemas en términos de sostenibilidad de sus pesquerías. Estos problemas no son de carácter técnico en el sentido de que los fundamentos, principios y elementos para una gestión sostenible de recursos pesqueros ya existen y los países que los adoptan han logrado varios grados de sostenibilidad en sus pesquerias. Los problemas de El Salvador en la pesca son debido a una inadecuada gobernanza pesquera y son de carácter político, cultural, y de educación.

Según Kooiman \& Bavinck (2005), Gobernanza es "el cuerpo completo de interacciones tanto públicas como privadas, implementadas para solucionar problemas y crear oportunidades. Incluye la formulación de principios que guíen tales interacciones y la consideración de las instituciones que se hagan cargo de los mismos". Usando esta definición, la gobernanza pesquera en El Salvador es inadecuada ya que no soluciona problemas en el sector pesquero y tampoco crea oportunidades para un desarrollo sostenible. E1 desarrollo de la pesca de atún sería una excepción a esta carencia de oportunidades 
de avances en las actividades pesqueras. Pero esta requiere un escrutinio por separado ya que no opera como otras pesquerías en el país. En términos de gobernanza pesquera, la transparencia y diálogo intersectorial son elementos indispensables para lograr formular principios acordados por los sectores que guíen las interacciones públicas y privadas y el comanejo de las actividades pesqueras. Además, la transparencia, diálogo intersectorial, mejora en los mecanismos que apoyan la inversión en ciencia y tecnología, darían un valor agregado a la gobernanza pesquera en El Salvador.

La inadecuada gobernanza pesquera es un problema de entendimiento general de lo que se requiere como país y de lograr un enfoque colectivo adecuado para solucionar problemas y crear oportunidades. Pero es un problema fundamentalmente de falta de voluntad política. El Estado debe dar la pauta para una buena gestión de los recursos pesqueros y continuar con la elaboración de una normativa que cumpla con el objetivo de su propia política. La inadecuada gobernanza pesquera es también un problema de innovación y de liderazgo a nivel político y a nivel científico. Existe muy poca coordinación entre los sectores, instituciones gubernamentales vinculadas, ONG y la cooperación internacional. Habiéndose oficialmente reconocido el potencial de la pesca para el bienestar social en El Salvador en múltiples ocasiones, y recientemente en la Política Nacional de Pesca y Acuicultura (MAG, 2015), la falta de voluntad política de los funcionarios en turno ha resultado en un continuo descuido y mal enfoque de CENDEPESCA. El Estado no juega el papel necesario para forjar buena gobernanza.

\section{2. ¿Cómo dimensionar la necesaria reforma institucional?}

Entendiendo el sistema pesquero como un trípode de componentes interactuantes e interdependientes (la política, la normativa y la institucionalidad), es importante recalcar que E1 Salvador tiene una nueva Política Nacional de Pesca y Acuicultura 2015-2030 y necesita adecuar la normativa vigente para que garantice los objetivos de dicha política. La ley de pesca vigente es la Ley General de Ordenación y Promoción de Pesca y Acuicultura (Decreto Legislativo n. 637 del 12/19/2001), con sus respectivas modificaciones. Esta ley precede la nueva política de pesca por 14 años y necesita ser adecuada y modernizada.

Actualmente existen dos anteproyectos de ley de pesca y acuicultura en la Asamblea Legislativa, uno que fue presentado como propuesta de los pescadores artesanales, y otro como propuesta de la Cámara Salvadoreña de Pesca y Acuicultura (CAMPAC). El anteproyecto de los pescadores artesanales/MAG fue elaborado por un consultor en el 2015, mucho tiempo previo a la promulgación de la nueva política. El anteproyecto de la CAMPAC es del 2017 y fue elaborado con el apoyo de la Secretaria para Asuntos Legislativos y Jurídicos de la Presidencia de la República. Pero también utilizó el anteproyecto del 2015 como base, y por eso tampoco considera la nueva Política Nacional de Pesca y Acuicultura como punto de partida. La nueva política tiene elementos nuevos que deben ser regulados normativamente, y los actuales anteproyectos de ley no los consideran. Como consecuencia, los objetivos, principios y ejes estratégicos de la nueva política de pesca 
no son atendidos debidamente en estas dos propuestas de ley. Es decir que la forma legal en la que se pretende conducir las actividades pesqueras (ley y reglamentos) de acuerdo a estos dos anteproyectos actualmente en la Asamblea Legislativa no refleja adecuadamente la nueva idea (la política 2015-2030, sus objetivos, principios y ejes estratégicos), la cual se ha acordado guiará al país hacia lograr pesquerías sostenibles y los otros objetivos de dicha política.

Un adecuado anteproyecto de ley deberá obligar a todos a actuar de acuerdo a los principios de la nueva Política Nacional de Pesca y Acuicultura 2015-2030, y deberá garantizar el cumplimiento de los objetivos estipulados en esta política. Es decir, todos los requisitos respectivos para lograr objetivos de acuerdo a los principios y ejes estratégicos de la política deberán ser regulados normativamente.

Teniendo en cuenta el objetivo específico, los principios y los ejes estratégicos planteados por la nueva Política Nacional de Pesca y Acuicultura 2015-2030, se debe redactar un nuevo anteproyecto de ley de tal forma que la institución rectora de regular las actividades pesqueras en El Salvador tenga la estructura debida y el enfoque requerido. Esto no se ha hecho todavía.

La pregunta que hay que responder no es “¿qué cambiar?", o "¿qué herramientas de gestión de cambio usar?”, sino “¿cómo cambiar lo que hay que cambiar?” para que el propósito trazado se logre. El propósito trazado aquí es de lograr un desempeño del Estado salvadoreño y de la sociedad tal que las pesquerías a futuro se desempeñen de forma sostenible, de acuerdo a la Constitución de la Republica y a los mejores intereses de la nación. El propósito trazado es lograr una buena gobernanza pesquera.

Viéndolo con un enfoque macro y desde el punto de vista de cómo lograr los cambios requeridos en el Gobierno para lograr pesquerías sostenibles en El Salvador, se proponen cinco factores que, directa o indirectamente, determinan la calidad de gestión de la institución oficial. Consideración de estos cinco factores será útil en la redacción de una nueva ley ya que un buen desempeño en cada factor contribuye a lograr y mantener pesquerías sostenibles. Una nueva ley tiene que garantizar pesquerías sostenibles a corto, mediano y largo plazo.

1. Autonomía administrativa y financiera.

2. Liderazgo político y científico.

3. Capacidad técnica, analítica y de investigación.

4. Funciones en el territorio.

5. Producción pesquera.

En términos de Autonomía administrativa y financiera, la administración de los recursos pesqueros requiere de un enfoque muy especializado donde debe predominar lo científico y tomar decisiones oportunas y efectivas. Esto no se puede lograr con la configuración actual de la institución rectora de la regulación delas actividades pesqueras en E1 Salvador. CENDEPESCA ha estado subyugada a las políticas administrativas y financieras del Ministerio de Agricultura y Ganadería por muchos años. También, de seguro, a manipulaciones políticas partidarias 
de las administraciones en turno a lo largo de su existencia. Como consecuencia de la carencia de autonomía administrativa y financiera no se ha logrado planificar a largo plazo y de acuerdo a las necesidades reales del sector. En años recientes, en la administración del presidente Funes por ejemplo, hubo cinco Directores de la institución. La nueva institución requiere de autonomía administrativa y de un presupuesto fijo y asegurado, por limitado que sea, enfocado en resolver problemas de carácter técnico/científico $\mathrm{y}$ así poder visualizar y ofrecer oportunidades de desarrollo multisectorial de acuerdo a la Política Nacional de Pesca y Acuicultura 2015-2030, la Constitución de la República y a los mejores intereses de la nación.

En términos de Liderazgo político y científico, y habiendo logrado autonomía administrativa y financiera, la institución rectora de la regulación de las actividades pesqueras en El Salvador necesita de un líder que defienda y dirija la línea de política de pesca y la formulación de planes de manejo de pesquerías. También se necesita de un líder científico que defina y dirija la línea técnica de investigación, análisis y diseminación de información y que asegure la incorporación debida de información científica en la elaboración de planes de manejo para todas las pesquerías del país. La ciencia es independiente, pero influenciada por la política en términos de prioridades de investigación, y la política está obligada a considerar los avances técnicocientíficos de acuerdo a los planes de manejo de pesquerías, a la Constitución de la República y a los mejores intereses de la nación.

En términos de Capacidad técnica, analítica y de investigación, la institución rectora de la regulación de las actividades pesqueras en $\mathrm{El}$ Salvador necesita garantizar el continuo flujo de información técnico-científica actualizada de forma transparente y con acceso al público. Asumiendo el objetivo de tener un plan de manejo para cada pesquería en el país, la formulación de planes de manejo, dentro de un marco de ordenación pesquera, requiere debate sobre el mérito de la información científica y acuerdos sobre su aplicabilidad en el co-manejo de pesquerías. Aparte de un liderazgo científico descrito arriba, se necesita de un programa de investigación propio de la institución. La cooperación científica de la institución con la academia, por ejemplo, podría permitir avances más rápidos, pero indiscutiblemente es la institución la que deberá garantizar que se cumplan los objetivos científicos propios del manejo de pesquerías. La mayoría de los elementos que se debaten públicamente entre los sectores afectados son de carácter técnicocientífico. La normativa pesquera deberá dictar como se debe usar esta información científica en la administración de actividades pesqueras. Y la toma de decisiones en cuestiones de carácter político (cuotas de pesca, restricción de acceso, etc), vigilada por la participación de los sectores de forma transparente, deberá obedecer a la Política Nacional de Pesca y Acuicultura vigente, a la Constitución de la República y a los mejores intereses de la nación.

En términos de Funciones en el territorio, la institución rectora de la regulación de las actividades pesqueras en El Salvador necesita orientar recursos en el territorio de acuerdo cúmulo total de requisitos necesarios para desarrollar, administrar y mejorar planes de manejo para todas las pesquerías de El 
Salvador. Asumiendo el objetivo de tener un plan de manejo para cada pesquería en el país, la organización territorial deberá ser diseñada para cumplir la tarea de desarrollar cada plan de manejo de pesquerías en un primer paso. En un segundo paso, la organización territorial deberá cumplir con lo requerido para administrar y mejorar cada uno y todos los planes de manejo de pesquerías de acuerdo a un Plan Nacional de Ordenación Pesquera, a la Constitución de la República y a los mejores intereses de la nación.

En términos de Producción pesquera, la institución rectora de la regulación de las actividades pesqueras necesita demostrar que todas las pesquerías de El Salvador, incluyendo pesquerías en aguas internacionales, son (o serán) sostenibles y se desarrollan dentro de planes de manejo basados en un marco de ordenación pesquera nacional de acuerdo a la Constitución de la República y a los mejores intereses de la nación.

\section{Conclusiones}

Lograr pesquerías sostenibles en El Salvador es equivalente a administrar un recurso público y patrimonio nacional regulando la actividad económica pesquera de tal forma que rinda beneficios a largo plazo y de acuerdo a la Constitución de la República. Lograrlo en este momento es principalmente cuestión de voluntad política y de darle a estos recursos su debida prioridad en la agenda nacional. Todas las herramientas, protocolos y conocimientos requeridos para lograr pesquerías sostenibles ya existen y se pueden adquirir y asimilar como país para lograr el objetivo trazado en la Política Nacional de Pesca y Acuicultura 2015-2030. El Estado debería continuar lo que comenzó con la promulgación de la Política Nacional de Pesca y Acuicultura 2015-2030. El siguiente paso es reestructurar la institución y darle su debido enfoque para lograr la capacidad necesaria para cumplir con el objetivo de esta nueva política. No hacerlo sería abandonar su propia política, independientemente de quien fue presidente cuando esta se promulgó.

Los cinco factores descritos arriba, o alguna modificación de estos como resultado de diálogo con los sectores, deberán ser considerados por un equipo interinstitucional para redactar, aprobar y luego implementar una nueva ley que reestructure a CENDEPESCA y así se garantice cumplir con el objetivo de la política nacional de acuerdo a sus principios y ejes estratégicos.

Se debería considerar crear una nueva entidad autónoma, y si fuese necesario tenerla adentro de un Ministerio, habría que considerar un cambio hacia el Ministerio de Economía. La pesca es una actividad económica. Habría que también evaluar cuidadosamente al personal actual y hacer los ajustes necesarios para minimizar conflictos laborales que podrían resultar por la falta de adaptación laboral a una nueva cultura institucional.

Habrá que mantener credibilidad en este proceso con una estrategia de logros a corto y mediano plazo, fomentando realmente un adecuado acompañamiento de las partes interesadas. Cambiar la estructura y funcionamiento de la CENDEPESCA y gradualmente aumentar su credibilidad ante el sector es una estrategia de logros a corto plazo. Lograr la capacidad de desarrollar e implementar planes de manejo para todas las pesquerías es una estrategia de logros a 
mediano plazo. Lo que se necesita es que todas las piezas relacionadas con la institucionalidad pesquera se conformen de tal forma que emerja una nueva y mejor gobernanza pesquera.

Pero a corto plazo habrá que mejorar la administración de los recursos pesqueros mientras se reforman las instituciones correspondientes y se logra la necesaria gobernanza para garantizar sostenibilidad en las pesquerías. A corto plazo, el uso de zonas exclusivas de pesca, el cooperativismo y el comanejo de los recursos pesqueros podrían ser los instrumentos más apropiados para lograr beneficios ecológicos, económicos y de alimentación. Esto ya se comenzó a trabajar en El Salvador, y solo habrá que continuar mejorándose en la práctica de acuerdo a una nueva y más acertada visión de país que realmente contribuya a formar mejor gobernanza.

\section{Referencias bibliográficas}

Costello, C., Ovando, D., Hilborn, R., Gaines, S.D., Deschenes, O., Lester, S.E. Status and solutions for the world's unassessed fisheries. Science. (2012); 338:517-520.

Kooiman, J., Bavinck, M. (2005). The governing perspective. In: Kooiman, J., et al. (Eds.), Fish for Life: Interactive Governance for Fisheries. Amsterdam University Press, Amsterdam, pp.11-24.

López, J. A. (2017). Presentación PowerPoint (línea del tiempo.pptx)

Ministerio de Agricultura y Ganadería. (MAG). (2015). Politica Nacional de Pesca y Acuicultura
2015-2030. San Salvador: Autor. Disponible en: http://extwprlegs1.fao.org/docs/pdf/els166099. pdf [Recuperado 5 Mar. 2018].

Ministerio de Agricultura y Ganadería (MAG). (2015). Plan Nacional de Ordenación de la Pesca y la Acuicultura. San Salvador: Autor.

Ministerio de Agricultura y Ganadería. MAG (2016). Plan Nacional de Desarrollo Sustentable de la Pesca y la Acuicultura en El Salvador 20152030. San Salvador: Autor.

Organización de Las Naciones Unidas Para la Agricultura y la Alimentación (FAO) (2004). Lineamientos para un Código de Ética de Pesca y Acuicultura para El Salvador. Basado en el trabajo de Alfredo García Mesinas. FishCode Revista No. 11. Roma.

Organización de Las Naciones Unidas Para la Agricultura y la Alimentación (FAO). (2005). Guía del Administrador Pesquero. Medidas de Ordenación y su Aplicación, Roma 2005. Roma: Autor. 